\title{
EXTRAÇÃO DE ÓLEO DE RESÍDUOS DE CARPA (Cyprinus carpio) E ANCHOITA (Engraulis anchoita)
}

\author{
J. O. GONÇALVES ${ }^{1}$, J. RIZZI ${ }^{1}$, R. S. POHNDORF ${ }^{1}$, F. R. SOBRAL ${ }^{1}$ e L. A. A. PINTO ${ }^{1}$ \\ ${ }^{1}$ Universidade Federal do Rio Grande, Escola de Química e Alimentos \\ E-mail para contato: jaques.rizzi@furg.br
}

\begin{abstract}
RESUMO - O aumento de resíduos de pescados gerados, e seu potencial uso para o reaproveitamento na geração de novos produtos, é uma importante preocupação industrial. Neste estudo foram analisadas as condições de operação para a obtenção de óleos de resíduos de carpa e de anchoita, pelo processo termomecânico de produção de farinha de pescado. Foi realizado um delineamento fatorial, sendo os fatores a trituração, o uso de vácuo, o tempo de extração e a centrifugação. Através dos perfis de ácidos graxos foram avaliadas as qualidades dos óleos obtidos. O maior rendimento na extração do óleo de vísceras de carpa (82\%), foi alcançado sem trituração, sob vácuo de $700 \mathrm{mmHg}$ durante 30 min e com centrifugação de $10000 \times$ g. A partir das melhores condições realizou-se dois tratamentos para a extração do óleo de resíduos de anchoita, em diferentes tempos de extração. O óleo obtido de vísceras de carpa apresentou valores de ácidos graxos saturados (AGS) de 31,2\%, e de insaturados (AGI) de 64,0\% (sendo 4,33\% de EPA+DHA), enquanto o óleo de resíduos de anchoita apresentou AGS de 38,0\% e AGI de $55,5 \%$ (sendo $31,0 \%$ de EPA+DHA).
\end{abstract}

\section{INTRODUÇÃO}

O crescimento da população e a procura por alimentos mais saudáveis tem impulsionado o aumento pela demanda no consumo de pescado nos últimos anos, desta forma a produção da pesca de captura e aquicultura, vem crescendo de forma significativa, o que aumenta os resíduos gerados pelas indústrias de beneficiamento (FAO, 2010). Os resíduos gerados no beneficiamento dos pescados (cabeça, vísceras, nadadeira, cauda, coluna vertebral, barbatana, escamas e restos de carne) representam aproximadamente $60-70 \%$ da matéria-prima utilizada, variando conforme as espécies e o tipo de processamento (Kolodziejska et al, 2008). Desta forma é necessário que as indústrias de processamento de pescados disponham de alternativas para o gerenciamento destes resíduos que venham a ser produzidos pelas indústrias. Esta preocupação torna-se um fator diferencial para as empresas, garantindo a diversificação da linha de produtos, crescimento sustentável e responsabilidade socioambiental (Bertoldi, 2003; Pessatti, 2001).

O aproveitamento dos resíduos de pescados através da obtenção de óleo bruto, utilizando processos termomecânicos de produção de farinha, é uma boa alternativa para evitar desperdícios e impedir a contaminação ambiental. Além disso, o óleo produzido pode ser utilizado não somente para a alimentação animal, mas também humana (Arvanitoyannis e Kassaveti, 2008). Óleos de pescado 


\section{9 a 22 de outubro de 2014 \\ Florianópolis/SC}

são considerados a principal fonte de ácidos graxos poli-insaturados ômega-3, especialmente o ácido eicosapentaenóico (EPA, 20:5) e o ácido docosahexaenóico (DHA, 22:6). Esses ácidos graxos têm destaque nas comunidades científicas e industriais devido aos benefícios à saúde humana, pois são essenciais para o desenvolvimento e funcionalidade de certos órgãos e para algumas respostas bioquímicas e fisiológicas do organismo (Fournier et al., 2007; Liu et al., 2006; Zhong et al., 2007).

As condições tradicionais de produção de farinha podem ocasionar ao óleo, transformações indesejáveis que afetam sua qualidade. Assim, o estudo das condições do processo termomecânico para obtenção de farinha e óleo torna-se importante, principalmente no que se refere à resíduos de pescado que contenham óleos mais nobres, para que o produto obtido mantenha suas características originais e gerem uma farinha de qualidade superior a tradicional. Algumas espécies apresentam em sua composição lipídica altas concentrações de ácidos graxos poli-insaturados, tornando a obtenção de óleo destas espécies mais interessante que a produção de farinha (Brody, 1965).

O presente trabalho teve por objetivo estudar diferentes condições de extração de óleo de resíduos de carpa (Cyprinus carpio) pelo processo termomecânico de produção de farinha de pescado, e utilizar as melhores condições para obtenção de óleo de resíduos de anchoita (Engraulis anchoita), avaliando o perfil graxo de ambos os óleos produzidos.

\section{MATERIAL E MÉTODOS}

\subsection{Matéria-prima}

As matérias-primas utilizadas foram resíduos de carpa (Cyprinus carpio) obtidos de uma planta comercial de processamento de pescado da cidade de Roca Sales/RS. Os resíduos de anchoita (Engraulis anchoita) foram coletados diretamente de uma indústria processadora de pescado da cidade de Rio Grande/RS. Ambos os resíduos foram acondicionados em monoblocos plásticos e transportados rapidamente até o Laboratório de Operações Unitárias/EQA/FURG/RS, onde foram armazenados a $-18^{\circ} \mathrm{C}$. Todos os demais reagentes utilizados eram de grau analítico.

\subsection{Procedimentos experimentais}

O óleo foi obtido pelo processo termomecânico de produção de farinha. Para todos os experimentos de extração do óleo bruto foram utilizadas condições análogas às de uma planta comercial, da indústria de processamento de pescado. Para elaboração da farinha de pescado, foram utilizados em torno de $0,45 \mathrm{~kg}$ de resíduos de cada espécie, por experimento realizado. Estes foram descongelados durante $12 \mathrm{~h}$ à temperatura ambiente. $\mathrm{O}$ procedimento foi realizado em escala de bancada, através das seguintes etapas: trituração, cocção, e centrifugação. Nos experimentos em que o material foi triturado, utilizou-se um triturador piloto (Metvisa Bing, Brusque-SC, Brasil). Após, foi realizada a produção da farinha em escala de bancada, para todos os experimentos referentes a matriz do delineamento experimental (Tabela 1), onde o material bruto passou por um processo de cocção, sob condições controladas, e posteriormente centrifugado à $25^{\circ} \mathrm{C}$ em centrífuga (Hitachi CR22GIII, Japão) para a separação das fases. $\mathrm{O}$ rendimento $(\mathrm{R})$ do óleo bruto foi calculado através das massas de 
óleo contidas na matéria prima e no material sólido após a extração.

\subsection{Métodos Analíticos}

O percentual de lipídios foi determinado através do método descrito por Bligh e Dyer (1959). Para os óleos brutos obtidos nas condições experimentais onde se obteve os melhores rendimentos, foi realizado o perfil graxo por análise cromatográfica. O preparo das amostras para a realização da análise cromatografia para determinação do perfil de ácidos graxos dos óleos foi realizado segundo metodologia descrita por Metcalfe e Schimitz (1966).

\subsection{Métodos Estatísticos}

Para avaliar os fatores que influenciaram na extração do óleo do resíduo da carpa foi utilizado um planejamento experimental fatorial completo $2^{4}$, considerando-se um nível de significância de $95 \%(\mathrm{p} \leq 0,05)$.

Na Tabela 1 estão apresentadas as variáveis de estudo utilizadas, com seus valores na forma real e na forma codificada. Estas variáveis (fatores de estudo) foram definidas por serem importantes no processo termomecânico para a produção de farinha de pescado.

Tabela 1- Matriz experimental com valores reais e na forma codificada.

\begin{tabular}{ccccccccc}
\hline \multirow{2}{*}{ Exp. } & \multicolumn{2}{c}{ Trituração } & \multicolumn{2}{c}{ Pressão } & \multicolumn{2}{c}{ Tempo (min) } & \multicolumn{2}{c}{ Rotação (× g) } \\
\cline { 2 - 9 } & A & A* & B & B* & C & C* & D & $\mathrm{D}^{*}$ \\
\hline 1 & s/trit. & -1 & s/vácuo & -1 & 20 & -1 & 7000 & -1 \\
2 & trit. & +1 & s/vácuo & -1 & 20 & -1 & 7000 & -1 \\
3 & s/trit. & -1 & c/vácuo & +1 & 20 & -1 & 7000 & -1 \\
4 & trit. & +1 & c/vácuo & +1 & 20 & -1 & 7000 & -1 \\
5 & s/trit. & -1 & s/vácuo & -1 & 30 & +1 & 7000 & -1 \\
6 & trit. & +1 & s/vácuo & -1 & 30 & +1 & 7000 & -1 \\
7 & s/trit. & -1 & c/vácuo & +1 & 30 & +1 & 7000 & -1 \\
8 & trit. & +1 & c/vácuo & +1 & 30 & +1 & 7000 & -1 \\
9 & s/trit. & -1 & s/vácuo & -1 & 20 & -1 & 10000 & +1 \\
10 & trit. & +1 & s/vácuo & -1 & 20 & -1 & 10000 & +1 \\
11 & s/trit. & -1 & c/vácuo & +1 & 20 & -1 & 10000 & +1 \\
12 & trit. & +1 & c/vácuo & +1 & 20 & -1 & 10000 & +1 \\
13 & s/trit. & -1 & s/vácuo & -1 & 30 & +1 & 10000 & +1 \\
14 & trit. & +1 & s/vácuo & -1 & 30 & +1 & 10000 & +1 \\
15 & s/trit. & -1 & c/vácuo & +1 & 30 & +1 & 10000 & +1 \\
16 & trit. & +1 & c/vácuo & +1 & 30 & +1 & 10000 & +1 \\
\hline
\end{tabular}

A,B,C,D correspondem aos valores reais; $A^{*}, B^{*}, C^{*} D^{*}$ correspondem aos valores codificados; s/trit: sem trituração; trit: trituração; s/vácuo: pressão atmosférica; c/vácuo: pressão absoluta de $60 \mathrm{mmHg} ;(\times \mathrm{g})$ : vezes força de gravidade. 


\section{RESULTADOS E DISCUSSÃO}

A Tabela 2 apresenta a matriz de planejamento experimental fatorial completo do tipo $2^{4}$ na forma codificada e as respostas obtidas para o percentual de rendimento em cada um dos experimentos.

Tabela 2 - Resultados da matriz do planejamento experimental fatorial, na forma codificada, para os percentuais de rendimento.

\begin{tabular}{cccccc}
\hline $\begin{array}{c}\text { Exp. } \\
\left(\mathrm{n}^{\mathbf{0}}\right)\end{array}$ & $\begin{array}{c}\text { Trituração } \\
\mathrm{A}^{*}\end{array}$ & $\begin{array}{c}\text { Pressão } \\
\mathrm{B}^{*}\end{array}$ & $\begin{array}{c}\text { Tempo } \\
\mathrm{C}^{*}\end{array}$ & $\begin{array}{c}\text { Rotação } \\
\mathrm{D}^{*}\end{array}$ & $\begin{array}{c}\text { Rendimento } \\
(\%)\end{array}$ \\
\hline 1 & -1 & -1 & -1 & -1 & $63,4 \pm 0,2$ \\
2 & +1 & -1 & -1 & -1 & $57,5 \pm 0,3$ \\
3 & -1 & +1 & -1 & -1 & $64,3 \pm 0,3$ \\
4 & +1 & +1 & -1 & -1 & $64,4 \pm 0,2$ \\
5 & -1 & -1 & +1 & -1 & $74,6 \pm 0,3$ \\
6 & +1 & -1 & +1 & -1 & $54,2 \pm 0,3$ \\
7 & -1 & +1 & +1 & -1 & $78,1 \pm 0,1$ \\
8 & +1 & +1 & +1 & -1 & $63,9 \pm 0,1$ \\
9 & -1 & -1 & -1 & +1 & $56,0 \pm 0,2$ \\
10 & +1 & -1 & -1 & +1 & $67,5 \pm 0,3$ \\
11 & -1 & +1 & -1 & +1 & $74,6 \pm 0,3$ \\
12 & +1 & +1 & -1 & +1 & $59,0 \pm 0,7$ \\
13 & -1 & -1 & +1 & +1 & $80,7 \pm 0,4$ \\
14 & +1 & -1 & +1 & +1 & $39,3 \pm 0,2$ \\
15 & -1 & +1 & +1 & +1 & $72,9 \pm 0,1$ \\
16 & +1 & +1 & +1 & +1 & $82,6 \pm 0,3$ \\
\hline
\end{tabular}

A análise de variância dos efeitos mostrou que para o percentual de rendimento (\%R), todos os efeitos principais trituração (A), vácuo (B), tempo (C) e rotação(D), bem como suas interações apresentaram significância ao nível de $95 \%$ de confiança $(\mathrm{p} \leq 0,05)$.

A Figura 1 apresenta os cubos de resposta dos experimentos para o rendimento (\% R), onde as arestas representam as variáveis e seus respectivos níveis, e nos vértices são apresentadas as respostas teóricas obtidas pela análise estatística. Observa-se que a região de trabalho onde se obteve os maiores rendimentos foi onde não ocorreu trituração do resíduo (nível -1), no maior tempo (nível +1), maior rotação (nível +1) e sob vácuo (nível +1). 


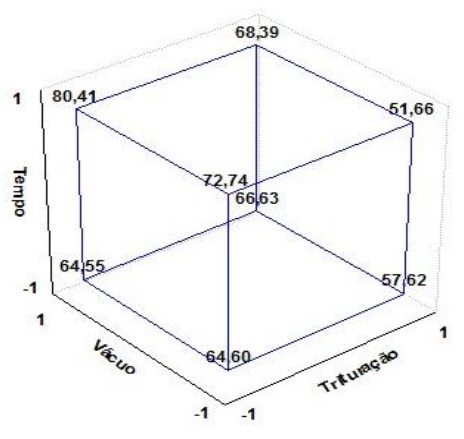

(a)

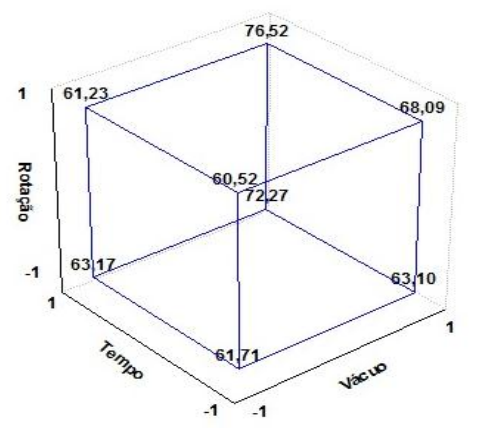

(c)

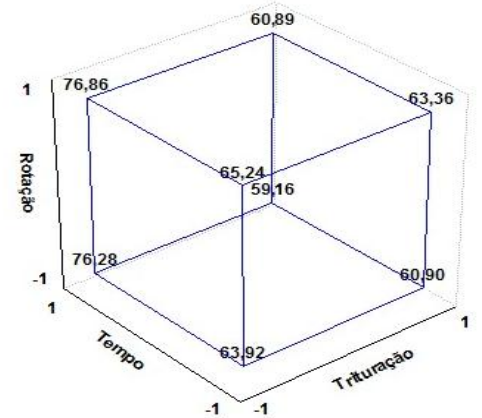

(b)

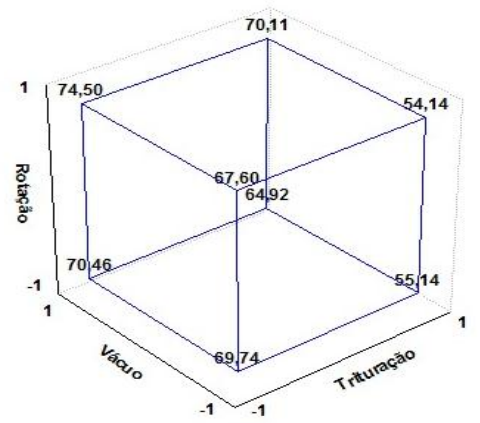

(d)

Figura 1 - Cubo de resposta para o rendimento (\%R) do óleo bruto extraído de carpa.

As análises cromatográficas foram realizadas nos experimentos que apresentaram maiores rendimentos, isto é, experimentos $\mathrm{n}^{\circ} 5, \mathrm{n}^{\circ} 7, \mathrm{n}^{\circ} 13$ e $\mathrm{n}^{\circ} 16$ da matriz de planejamento experimental fatorial (Tabela 2). Sendo que estes apresentaram percentuais de rendimento de 74, 78, 80 e $82 \%$ respectivamente. Assim, para alcançar o maior rendimento de óleo de carpa (82\%), os resíduos não devem ser triturados e a extração deve ser feita sob vácuo de $700 \mathrm{mmHg}$, utilizando um tempo de 30 min, e a centrifugação para separação do óleo de 10000 ×g.

A Tabela 3 apresenta o perfil graxo (\%) dos óleos brutos de carpa, proveniente dos experimentos $\mathrm{n}^{\circ} 5, \mathrm{n}^{\circ} 7, \mathrm{n}^{\circ} 13$ e $\mathrm{n}^{\circ} 16$. Observa-se que, os maiores percentuais de ácidos graxos identificados nos óleos de carpa são C18:1+C18:3 (oléico e linolênico), C16:0 (palmítico), C16:1 (palmitoléico), C18:2 (linoléico), C18:0 ( esteárico), constituindo aproximadamente 85\% dos óleos.

Os peixes sintetizam EPA e DHA e ácido araquidônico (AA), pois o alongamento e capacidade de dessaturação do ácido $\alpha$-linolênico (18:3) são insuficientes para satisfazer as necessidades dos mesmos (Miller et al., 2007). Na Tabela 3, observa-se que os conteúdos de DHA e de AA nos óleos de carpa provenientes dos experimentos são maiores que os citados por Crexi et al., (2009), de 1,24\%, $1,20 \%$ respectivamente. Porém, o conteúdo de EPA é menor que o citado pela autora $(3,82 \%)$. A causa para esta variação nos resultados observados é devido, possivelmente à alimentação destes peixes. As variações na composição de ácidos graxos podem estar relacionadas por mudanças nos hábitos nutricionais dos peixes (Arts et al., 2001; De Silva et al., 1997; Borlongan e Benitez, 1992). 
Tabela 3 - Perfil graxo dos óleos obtidos nos experimentos de maior rendimento para óleo de resíduo de carpa.

\begin{tabular}{|c|c|c|c|c|}
\hline Ácidos graxos & $\begin{array}{c}\text { Experimento } \\
\text { n}^{\circ} 5(\%)\end{array}$ & $\begin{array}{c}\text { Experimento } \\
\mathrm{n}^{\circ} 7(\%)\end{array}$ & $\begin{array}{c}\text { Experimento } \\
\mathrm{n}^{\circ} 13(\%)\end{array}$ & $\begin{array}{c}\text { Experimento } \\
n^{\circ} 16(\%)\end{array}$ \\
\hline $\mathrm{C} 12: 0$ & - & $0,22 \pm 0,01^{\mathrm{a}}$ & - & - \\
\hline C14:0 & $2,55 \pm 0,16^{\mathrm{a}}$ & $3,70 \pm 0,17^{\mathrm{a}}$ & $2,91 \pm 0,23^{\mathrm{a}}$ & $3,73 \pm 0,01^{\mathrm{a}}$ \\
\hline $\mathrm{C} 15: 0$ & - & $1,14 \pm 0,06^{\mathrm{a}}$ & $0,89 \pm 0,09^{\mathrm{a}}$ & $1,13 \pm 0,02^{\mathrm{a}}$ \\
\hline C16:1 & $6,46 \pm 0,25^{\mathrm{a}}$ & $7,44 \pm 0,21^{\mathrm{a}}$ & $7,00 \pm 0,27^{\mathrm{a}}$ & $7,86 \pm 0,03^{\mathrm{a}}$ \\
\hline C16:0 & $21,86 \pm 0,28^{a}$ & $20,08 \pm 0,28^{a}$ & $19,63 \pm 0,29^{a}$ & $21,77 \pm 0,43^{\mathrm{a}}$ \\
\hline C17:0 & - & $0,59 \pm 0,05^{\mathrm{a}}$ & $0,79 \pm 0,04^{\mathrm{a}}$ & - \\
\hline C18:2 & $8,86 \pm 0,19^{a}$ & $7,21 \pm 0,12^{\mathrm{a}}$ & $10,70 \pm 0,07^{\mathrm{a}}$ & $7,50 \pm 0,08^{\mathrm{a}}$ \\
\hline $\mathrm{C} 18: 1 \mathrm{c}+\mathrm{C} 18: 3$ & $39,98 \pm 1,31^{\mathrm{a}}$ & $37,28 \pm 1,12^{a}$ & $38,19 \pm 1,17^{\mathrm{a}}$ & $39,0 \pm 0,70^{\mathrm{a}}$ \\
\hline C18:1t & $3,97 \pm 0,16^{\mathrm{a}}$ & $3,16 \pm 0,11^{\mathrm{a}}$ & - & - \\
\hline C18:0 & $7,01 \pm 0,16^{\mathrm{a}}$ & $4,49 \pm 0,03^{\mathrm{a}}$ & $6,13 \pm 0,11^{\mathrm{a}}$ & $6,27 \pm 0,06^{\mathrm{a}}$ \\
\hline C20:4 (AA) & $1,86 \pm 0,06^{\mathrm{a}}$ & $1,74 \pm 0,04^{\mathrm{a}}$ & $1,53 \pm 0,02^{\mathrm{a}}$ & $1,77 \pm 0,02^{\mathrm{a}}$ \\
\hline C20:5 (EPA) & $1,94 \pm 0,05^{\mathrm{a}}$ & $2,91 \pm 0,02^{\mathrm{a}}$ & $1,99 \pm 0,01^{\mathrm{a}}$ & $2,67 \pm 0,01^{\mathrm{a}}$ \\
\hline C20:3 & - & $0,52 \pm 0,03^{\mathrm{a}}$ & - & - \\
\hline C20:1 & - & $1,62 \pm 0,06^{\mathrm{a}}$ & $1,88 \pm 0,04^{\mathrm{a}}$ & $1,99 \pm 0,04^{\mathrm{a}}$ \\
\hline C20:0 & - & - & - & - \\
\hline 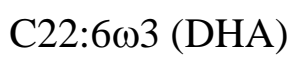 & $2,06 \pm 0,02^{\mathrm{a}}$ & $1,97 \pm 0,03^{\mathrm{a}}$ & $1,67 \pm 0,01^{\mathrm{a}}$ & $2,12 \pm 0,02^{\mathrm{a}}$ \\
\hline $\mathrm{C} 22: 5$ & - & $0,40 \pm 0,12^{\mathrm{a}}$ & $0,69 \pm 0,03^{\mathrm{a}}$ & - \\
\hline Total $(\%)$ & $96.55 \pm 1,21^{\mathrm{a}}$ & $90,85 \pm 1,55^{\mathrm{a}}$ & $94,00 \pm 0,96^{\mathrm{a}}$ & $95,80 \pm 1,05^{\mathrm{a}}$ \\
\hline$\sum \mathrm{ni}^{* *}$ & 3,45 & 6,15 & 6,00 & 4,22 \\
\hline
\end{tabular}

Utilizando as melhores condições definidas no planejamento experimental (s/trituração, uso de vácuo e centrifugação de $1000 \times \mathrm{g}$ ), foram realizados dois tratamentos para extração de óleo tendo como matéria-prima os resíduos de anchoita, nos tempos de 20 e 30 min. Os ácidos graxos mais encontrados no óleo de anchoita, constituindo cerca de 87,5\% do óleo, foram C14:0 (mirístico), C16:1 (palmitoléico), C16:0 (palmítico), C18:1+C18:3 (oléico e linolênico), C18:0 (esteárico), EPA e DHA.

Assim como foi observado no óleo de carpa, os ácidos graxos saturados (AGS) mais representativos no óleo de anchoita foram o ácido palmítico (C16:0), seguido pelo ácido mirístico (C14:0) e pelo esteárico (C18:0). O aumento do tempo de extração de 20 para 30 min, proporcionou elevação no percentual de $\mathrm{C} 18: 1 \mathrm{c}+\mathrm{C} 18: 3$ de 12,66\% para 14,3\% e uma diminuição em C22:6 63 (DHA) de 24,7\% para 21,4\%, respectivamente. Para o óleo de anchoita, o total de AGS ficou em 


\section{9 a 22 de outubro de 2014 \\ Florianópolis/SC}

torno de $38 \%$, valor superior ao óleo de carpa que apresentou um percentual em torno de $31,2 \%$. O somatório de ácidos graxos insaturados (AGI), aproximadamente 55,5\%, foi inferior ao encontrado no óleo de carpa, porém a quantidade média de EPA+DHA (31\%) foi em torno de sete vezes maior que o de carpa. Este valor é muito superior ao encontrado no óleo de corvina (11,5\%), um pouco superior ao óleo de sardinha (25-30\%) e inferior ao óleo de albacore (36,7\%), valores estes da literatura (Monte el al., 2012; Brody, 1965).

\section{CONCLUSÕES}

Para a extração do óleo bruto de resíduos de carpa, através do processo termomecânico de obtenção de farinha observou-se, através de análise estatística, que para um maior rendimento do óleo obtido, os resíduos não devem ser triturados e a extração deve ser feita sob vácuo de $700 \mathrm{mmHg}$, sendo o tempo de extração de 30 min e a centrifugação de 10000 ×g.

Os maiores conteúdos de ácidos graxos identificados nos óleos de carpa obtidos nos experimentos foram palmítico, palmitoléico, esteárico, oléico, linoléico e linolênico, constituindo aproximadamente $85 \%$ dos óleos. O teor de ácidos graxos saturados (AGS) foi em torno de 31,2\%, sendo o palmítico o AGS mais abundante. Os conteúdos de ácidos graxos insaturados (AGIs) dos óleos obtidos variaram de $62,9 \%$ a $65,1 \%$.

Na extração de óleo de anchoita, não houve diferença significativa $(\mathrm{p} \leq 0,05)$ para os dois tempos de extração utilizados. Os ácidos graxos mais encontrados no óleo de anchoita foram mirístico, palmítico, palmitoléico, esteárico, oléico, linolênico, EPA e DHA, e estes constituiram em torno de $87,5 \%$ do óleo. O somatório de AGIs foi em torno de 55,5\%, sendo que os valores de EPA+DHA corresponderam ai aproximadamente $31,0 \%$. Desta forma foi possível verificar que as condições estabelecidas anteriormente, para a extração de óleo de resíduos de anchoita pelo processo termomecânico de obtenção de farinha, preservaram as características do óleo.

\section{REFERÊNCIAS}

ARTS, M.T.; ACKMAN, R.G; HOLUB, B.J. 'Essential fatty acids' in aquatic ecosystems: a crucial link between diet and human health and evolution. Can. J. Fish.Aquat. Sci., v.58, p.122-137, 2001.

ARVANITOYANNIS, I.S.; KASSAVETI, A. Fish industry waste: treatments, environmental impacts, current and potential uses. Int. J. Food Sci. Technol., v.43, p.726-745, 2008.

BERTOLDI, F. C. Efeito do Lactobacillus casei subsp. casei ATCC 393 na redução do sabor amargo da carne escura de atum. Florianópolis: UFSC, 2003. 61p. Dissertação Mestrado.

BLIGH, E.G.; DYER, W.J. A rapid method of total lipid extraction and purification. Can. J. Biochem. Physiol., v.27, p.911-917, 1959.

BORLONGAN, I.G.; BENITEZ, L.V. Lipids and fatty acid composition of milkfish (Chanos chanos Forsskal) grown in freshwater and seawater. Aquaculture, v.104, p.79-89, 1992.

BRODY, J. Fisheries by-products technology. The AviPublishing, 1965. 
CREXI, V.T.; MONTE, M. L.; SOUZA-SOARES, L. A.; PINTO, L. A. A. Production and refinement of oil from carp (Cyprinus carpio) viscera. Food Chem., v.119, p.145 - 150, 2010.

DE SILVA, S.S.; GUNASEKERA, R.M.; AUSTIN, C.M. Changes in fatty acid profile of hybrid red tilapia Oreochromismossambicus $X$ O. niloticus, subjected to short-term starvation and comparison with changes in seawater raised fish. Aquaculture, v.153, p.273-290, 1997.

FOOD AND AGRICULTURE ORGANIZATION OF THE UNITED NATIONS (FAO). 2010. The state of world fisheries and aquaculture. Disponível em: http://www.globefish.org/fish-oil-november-2009.html. Acesso em 05 maio. 2012.

FOURNIER, V.; DESTAILlATS, F.; HUG, B.; GOLAY, P. A., JOFFRE, F.; JUANEDA, P. Quantification of eicosapentaenoic and docosahexaenoic acid geometrical isomers formed during fish oil deodorization by gas-liquid chromatography. J. Chromatogr. A, v.1154, p.353-359, 2007.

KOLODZIEJSKA, I; SKIERKA, E; SADOWSKA, M; KOLODZIEJSKI, W; NIECIKOWSKA, C. Effect of extracting time and temperature on yield of gelatin from different fish offal. Food Chem., v.107, p.700-706, 2008.

LIU, S.; ZHANG, C.; HONG, P.; JI, H. Concentration of docosahexaenoic acid (DHA) of tuna oil by urea complexation: optimization of process parameters. J. Food Eng., v.73, p.203209, 2006.

METCALFE, L.D.A.A.; SCHIMITZ, J.R. Rapid preparation of fatty acid esters from lipids for gas liquid chromatography. Analytical Chem., v.38, p.510, 1966.

MILLER, M.R.; NICHOLS, P.D.; CATER, C.G. Replacement of fish oil with thraustochytrid Schizochytrium sp. L oil in Atlantic salmon parr (Salmo salar L) diets. Comp. Biochem. Physiol., v.148, p.382-392, 2007.

MONTE, M. L.; PAIM, R. M.; RIZZI, J.; TAVARES, J. L. S.; PINTO, L. A. A. Obtenção de óleo de pescado com alto valor agregado a partir do aproveitamento de resíduos de corvina e anchoita. In: XIX Congresso Brasileiro de Engenharia Química - COBEQ, 2012, Búzios RJ.

PESSATTI, M. L. Aproveitamento dos sub-produtos do pescado. Itajaí: MAPA/UNIVALI, 2001. 130p.

ZHONG, Y.; MADHUJITH, T.; MAHFOUZ, N.; SHAHIDI, F. Compositional characteristics of muscle and visceral oil from steelhead trout and their oxidative stability. Food Chem., v.104, p.602-608, 2007. 